\title{
The role of MRI-CSF flowmetry in the diagnosis of idiopathic normal pressure hydrocephalus
}

\author{
Ashraf Talaat Youssef, Ahmed M. Magdy, Amal Abdul-Aleem Abdul-Rahman
}

\begin{abstract}
:
Background: Normal pressure hydrocephalus is a gloomy entity with no definite cause known till date. The classical clinical triad includes gait apraxia, urinary incontinence and different grades of cognitive dysfunction as dementia. NPH is considered the first treatable type of dementia as it differs from other dementias in that the symptoms can show clinical recovery \& regression with CSF diversion.
\end{abstract}

Many tests have been employed in the diagnosis of idiopathic NPH, including invasive methods which may lead to serious complications. That is why; non-invasive techniques are required to increase the sensitivity and specificity of routinely used imaging methods.

Flow sensitive cardiac gated phase contrast MR imaging techniques have been increasingly applied recently providing the potential for non-invasive study of CSF flow dynamics, as it can be used to discriminate between different types of hydrocephalus \& to provide significant information in preoperative evaluation of $\mathrm{NPH}$ and the prediction of the benefit from surgery.

The aim of this study: is to evaluate the role of MRICSF flowmetry using its various flow indices for the diagnosis of INPH.

Patients and methods: In our prospective study conducted at radiology department in our institute including thirty cases: 25 patients with clinically suspected NPH \& ventriculomegaly \& 5 normal elderly control cases.

Two protocols of PC MRI CSF flowmetry examination were used, one assessing CSF flow dynamics qualitatively using a sagittal plane and one

\section{Introduction:}

NPH was first described by Hakim and Adams in 1965 as a syndrome of gait apraxia, dementia and incontinence associated with normal opening CSF pressure and dilated ventricles ${ }^{(1)}$. quantitatively using an axial plane perpendicular to the aqueduct.

Results: Among the 25 patients involved in the study, 19 patients $(76 \%)$ were diagnosed as NPH based on the clinical \& radiological findings as well as the hyper dynamic CSF flow across the aqueduct in the PC MRI study \& 6 patients (24\%) were diagnosed as atrophic dilatation with hypo dynamic CSF flow across the aqueduct in the PC MRI study.

In our study, we found that normal CSF flow throughout the cardiac cycle show free aqueductal pulsatile CSF flow as a cranial flow during CSF diastole (hyper intense signal) and caudal flow during systole (hypo intense signal).

In our study, we found that at a cut off value for aqueductal SV of about $42 \mathrm{ul} /$ cycle. Aqueductal stroke volume (> 42) shows sensitivity of about $(93.3 \%) \&$ specificity of about (75\%) with total accuracy of about $(89.5 \%)$ in predicting shunt responsiveness in NPH patients.

\section{Conclusion:}

Phase contrast MRI CSF Flowmetry was found to be of high sensitivity and specificity in diagnosing NPH \& differentiating it from atrophic dilatation. It is simple, fast and non-invasive effective method that adds more to the total accuracy of the conventional MRI examination as it provide valuable additional information \& reducing rates of complications and predicting shunt responsiveness.

Key words: Normal pressure hydrocephalus, ventriculomegaly, CSF Flowmetry, Phase contrast MRI.

The incidence of NPH is much higher in elderly populations; about 49.3 per 100,000 for people 60 to 69 years of age. The majority of cases are idiopathic. The diagnostic challenge in the management of each case is to differentiate between NPH and involutional brain changes with overlapping symptoms in such age group 
and to balance the risks involved with surgery in this age group against the potential benefits of treating the component that may be remedied by CSF diversion ${ }^{(2)}$.

The underlying cause remains controversial. A theory suggests that NPH is considered a form of CSF circulation disorder with loss of elasticity \& compliance of ventricular walls and subsequent pressure gradient between the ventricles and periventricular tissues favoring progressive ventriculomegaly; the ventricular expansion may distort the deep white matter connections and/or blood vessels between the basal ganglia and frontal cortex responsible for producing the characteristic symptoms (Adam's / Hakim's triad) (3).

Many tests have been employed in the diagnosis of INPH, including invasive methods which may lead to serious complication. That is why; flow sensitive cardiac gated phase contrast MR imaging techniques have been increasingly applied providing the potential for non-invasive study of CSF flow dynamics ${ }^{(4)}$.

CSF flow MRI can be used to discriminate between communicating and noncommunicating hydrocephalus, to localize the level of obstruction in obstructive hydrocephalus $\&$ to provide significant information in preoperative evaluation of $\mathrm{NPH}$, differential diagnosis and the prediction of the benefit from surgery and post-operative follow-up ${ }^{(5)}$.

On imaging; it can be characterized both on CT and MRI by enlarged lateral and third ventricles out of proportion to the cortical sulcal enlargement; yet not diagnostic in cases of NPH. Evans' index value (more than 0.3 ) is considered one of the diagnostic guidelines in NPH; which depends on the ratio of maximum width of the frontal horns of the lateral ventricles and maximal internal diameter of skull at the same level employed in axial CT and MRI images. Unfortunately; it is a very rough marker of ventriculomegaly and varies greatly depending on the location and angle of the slice ${ }^{(6)}$.
Therefore; imaging techniques are increasingly developing \& leading to greater access to the visualization of physiological processes and structural details as well as diagnostic tests and prognostic tools for assessment of patients with suspected $\mathrm{NPH}^{(7)}$.

MRI-CSF flow studies using cine phase contrast MRI imaging is a promising, safe and non-invasive investigation for quantitative and qualitative CSF flow analysis to be used for the diagnosis of normal pressure hydrocephalus especially in those cases where the differentiation from atrophy on clinical and conventional radiological basis is difficult. Increased CSF stroke volume through the aqueduct has been demonstrated in the NPH population despite normal CSF pressures ${ }^{(\mathbf{8})}$.

Aqueductal CSF stroke volume was compared with surgical post shunting results and noted that the degree of improvement was better in patients with higher preoperative stroke volume; allowing MR-CSF flowmetry to be a promising diagnostic and prognostic tool ${ }^{(9)}$.

CSF stroke volume has a role in selection of patients into shunt responders \& less likely responding but still improving on shunt procedures; yet should not be used as exclusion criteria $^{(\mathbf{1 0})}$.

\section{Materials and Methods:}

The study was carried on 25 patients (group I) suspected to have NPH based on clinical symptoms and conventional MRI findings and 5 normal healthy elderly control persons (group II).

Inclusion criteria: Patients with clinical symptoms suspicious of NPH including Hakim triad together with dilated ventricular system.

\section{Exclusion criteria}

- Patients with routine MRI contraindication as peacemaker, claustrophobic patients, intracranial metal clips and neuro-stimulators. 
- Patients having secondary causes for NPH on imaging as subarachnoid bleeding, tuberculoma meningitis, cranial traumas or intra-cranial surgery.

Written informed consents were obtained from all patients after full explanation of the technique and how the machine work.

There was a code number for each patient's file that includes all investigations. Privacy of all patients' data was guaranteed.

Any unexpected risks that appeared during the course of the research were cleared to the participants and the ethical committee on time.

\section{Methods:}

All patients were evaluated by:

1. Complete history taking including present and past history.

- Personal history: as regards the name, age and gender.

- Present history: as regards the presenting symptoms like headache, tinnitus, dizziness, gait disturbance, dementia and urinary incontinence as well as the duration of illness.

- Past history: as regards previous surgical intervention, stroke, cranial traumas or previous history of meningitis.

MRI examinations were performed with 1.5 T MR Unit (Toshiba Vantage Titan closed machine), Imaging was carried out using standard circular polarized head-array coils and ultragradients, in neutral supine position and without any case preparation. Patients were asked to avoid deep breathing during the examination.

All patients' results were correlated with a diagnostic lumbar puncture manometry and lumbar tap test.
The diagnosis of NPH was based upon the clinical \& radiological findings as well as the presence of hyper dynamic CSF flow on PC MRI.

Diagnosed patients with NPH were followed up after shunting surgery at neurosurgery \& neurology outpatient clinics by following up the improvement of clinical symptoms \& categorized into well responsive to shunt or not.

\section{Results:}

Our study was conducted in our institute including thirty cases: 25 patients with clinically suspected NPH \& ventriculomegaly \& 5 normal elderly control cases.

Among the 25 patients involved in the study, 19 patients $(76 \%)$ were diagnosed as NPH based on the clinical \& radiological findings as well as the hyper dynamic CSF flow across the aqueduct in the PC MRI study \& 6 patients (24\%) were diagnosed as atrophic dilatation with hypo dynamic CSF flow across the aqueduct in the PC MRI study.

\section{Demographic data:}

Socio-demographics: The mean age of NPH group was about $(63.7 \pm 7.2)$, the mean age of the atrophic dilatation group was about $(71.0 \pm 3.2)$ $\&$ for the control group was about $(55.8 \pm 3.3)$

Table (1).

\section{Analysis of NPH symptomatology:}

- Dementia: Seventeen of nineteen patients of $\mathrm{NPH}$ had dementia as a presenting symptom $(89.5 \%)$.

- Urine incontinence: Nine of nineteen patients had urine incontinence (47.4\%) mostly associated with dementia or gait disturbance as it is considered to be late symptom of NPH. 
- Gait disturbance: Fifteen of nineteen patients of NPH had gait disturbance $(78.9 \%)$ as a presenting symptom or associated with other symptoms of the Hakim's triad.

Important finding of conventional MRI in NPH patients:

- Ventricular dilatation: All patients of NPH had dilated lateral $\& 3^{\text {rd }}$ ventricles \pm $4^{\text {th }}$ ventricle $(100 \%)$.

- Aqueductal flow void: Fifteen of nineteen patients of NPH had pronounced flow void noted at the aqueduct $\pm 3^{\text {rd }} \&$ $4^{\text {th }}$ ventricle $(78.9 \%)$.

- Associate brain ischemic changes or temporal lobe atrophy: Three of nineteen patients of NPH had associated ischemic changes or temporal lobe atrophy $(15.3 \%)$.

Differences in PSV and Aq. Stroke volume according to study groups:

\section{As regards PSV:}

- $\mathbf{N P H}$ vs Control: (Mean $=10.4$ vs 5.4 $\mathrm{cm} / \mathrm{s}$ ) was significantly increased in NPH with statistically significant difference $(\mathrm{P}$ value of about 0.020 ).

- AD vs Control: (Mean $=4.3$ vs $5.4 \mathrm{~cm} / \mathrm{s}$ ) was reduced in AD with statistically nonsignificant difference ( $\mathrm{P}$ value of about 1.000).

As regards Aqueductal stroke volume:

- $\quad$ NPH vs Control: $($ Mean $=109.5$ vs 27.6 microliter/cycle) was significantly increased in NPH with statistically significant difference $(\mathrm{P}$ value of about 0.015).

- AD vs Control: $($ Mean $=16.2$ vs 27.6 microliter/cycle) was reduced in $\mathrm{AD}$ with statistically non-significant difference ( $\mathrm{P}$ value of about 1.000).
Differences in PSV and Aq. Stroke volume according to study groups: PSV \& Aqueductal stroke volume were significantly increased in NPH patients Table (2).

Differences in PSV and Aq. Stroke volume in relation to shunt responsiveness in NPH patients:

- As regards PSV: 15 of 19 patients had well response to shunt with mean PSV of about $(11.4 \pm 3.5 \mathrm{~cm} / \mathrm{s})$, the four $\mathrm{NPH}$ shunt non responsive cases had mean PSV of about $(6.6 \pm 1.8 \mathrm{~cm} / \mathrm{s})$.

- As regards Aqueductal Stroke volume: The 15 patients who had well shunt response, mean SV was about (125.9 \pm 67.4 microliter/cycle), while the $4 \mathrm{NPH}$ shunt non responsive cases had mean $\mathrm{SV}$ of about (48 $\pm 21.4 \mathrm{ul}$ )/cycle. Table (3)

Relation between Aq. Stroke volume (> 42) and shunt responsiveness in NPH patients: Table (4):

- SV> 42: Fourteen out of fifteen patients who had well response to shunt operation (93.3\%) had SV above 42.

- $\mathbf{S V}=$ 28-42: Three out of four patients who had not responded to shunt operation (75\%) had SV between (28-42).

One NPH shunt responsive patient had SV between (28-42) suggestive of mild hyper dynamic CSF flow \& early NPH with positive LTT, this mean that SV above 42 favors good outcome after shunt operation \& SV between (28-42) is not an exclusion criteria for shunt responsiveness. (Figure $1 \& 2$ ).

One NPH shunt non-responsive patient had SV above 42 with negative LTT, which is explained by the concomitant evident brain ischemic changes \& temporal lobe atrophy.(Figure 3 \& 4). 
Prognostic accuracy of Aq. Stroke volume (> 42) in predicting shunt responsiveness in NPH patients:

- Aqueductal stroke volume (> 42) shows sensitivity of about $(93.3 \%) \&$ specificity of about (75\%) with total accuracy of about $(89.5 \%)$ in predicting shunt responsiveness in NPH patients.

\section{Relation between LTT and shunt responsiveness in NPH patients:}

- Fourteen out of fifteen patients who had well response to shunt operation had positive LTT (93.3\%).

- Four patients who not responded to shunt were all negative to LTT $(100 \%)$.

One NPH shunt responsive patient had negative LTT with hyper dynamic CSF flow in PC MRI suggesting that negative LTT alone is not an exclusion criteria.

N.B: The six patients diagnosed with AD had negative LTT.

\section{Relation between LTT and shunt responsiveness in NPH patients:}

- 14 out of 15 patients who had well response to shunt operation had positive LTT $(93.3 \%)$.

- 4 patients who not responded to shunt were all negative to LTT (100\%).

One NPH shunt responsive patient had negative LTT with hyper dynamic CSF flow in PC MRI suggesting that negative LTT alone is not an exclusion criteria. Table (5)

\section{Prognostic accuracy of LTT in predicting shunt responsiveness in NPH patients:}

- $\quad$ LTT shows sensitivity of about (93.3\%) \& specificity of about (100\%) with total accuracy of about (94.7\%) in predicting shunt responsiveness in NPH patients.

\section{Discussion:}

The diagnosis of NPH was based upon the clinical \& radiological findings as well as the presence of hyper dynamic CSF flow on PC MRI.

Diagnosed patients with NPH were followed up after shunting surgery at neurosurgery \& neurology outpatient clinics by following up the improvement of clinical symptoms \& categorized into well responsive to shunt or not.

We investigated CSF flow characters in the three groups. Two imaging planes were applied: one in the sagittal plane with plane velocity encoded in the caudo-cranial direction for a qualitative assessment $\&$ one in the axial plane with through-plane velocity encoded in the caudo-cranial direction for flow quantification.

Qualitative assessment of CSF flow: (sagittal phase images) (static and dynamic cine display):

In our study, we found that normal CSF flow throughout the cardiac cycle show free aqueductal pulsatile CSF flow as a cranial flow during CSF diastole (hyper intense signal) and caudal flow during systole (hypo intense signal).

Results of our study support the current opinion of CSF circulation that was indeed described by Bhadelia RA et al ${ }^{(11)}$, that the cardiac cycle-related cerebral blood volume variations produce bi-directional oscillatory movement of CSF within the cranio-spinal axis. During systole, the net inflow of blood increases the intracranial volume and induces cranio-caudal flow (hypo intense). During diastole, the net outflow of blood decreases the intracranial volume and promotes caudo-cranial flow (hyper intense) ${ }^{(\mathbf{1 2})}$ also reported the same finding.

Rovira A et al stated that Consistent pulsatile pattern of to-and-fro movements of flow in the aqueduct, foramen of Magendie, and in the 
dorsal and ventral subarachnoid space at the cervico-medullary junction was found ${ }^{(\mathbf{1 3})}$. It was observed in our normal volunteers and patients.

In our study flow void phenomenon was not clearly visualized in healthy volunteers. Schroder H.W.S. et al, stated that the visualization of this effect on routine $\mathrm{T} 1$ or T2WI was not consistent ${ }^{(\mathbf{1 4})}$. However on sagittal T2WI turbo inversion recovery MR images, they could depict this sign in most healthy volunteers.

Our study also showed that flow void phenomenon is most pronounced in 15 out of 19 $\mathrm{NPH}$ diagnosed patients with a percent of about (78.9\%) and is not evident in patients with atrophy.

Schroth G. and Klose, reported the same finding, \& also found that patients with brain atrophy have hardly any flow void in the aqueduct ${ }^{(15)}$.

\section{Quantitative assessment of CSF flow (axial phase contrast images):}

In our study, All healthy volunteers and patients revealed aqueductal cranial flow during CSF diastole (hyper intense signal) and caudal flow during CSF systole (hypo intense signal). The CSF waveforms generated were similar. Various parameters, i.e., time, velocity, and flow, have been used to examine CSF flow dynamics.

\section{In the control group:}

In our study, the peak systolic velocity (PSV) at the level of the aqueduct was around $5.4( \pm 0.3) \mathrm{cm} / \mathrm{s}$, systolic stroke volume (SV) was around $27.6( \pm 2.3)$ microliter/cycle, which is very close to the study reported by Schroder H.W.S et al (14) who reported a SV of 28 microliter in normal individuals

Ihab I. Baker et al also mentioned that, in the control group, the PSV was around 5.25 $\mathrm{cm} / \mathrm{sec}( \pm 1.67)$. A relatively wide range of normal aqueductal CSF velocity values measured by the cine-phase contrast and mean value of 30
$( \pm 9.8)$ microliters for the aqueductal systolic SV in healthy volunteers ${ }^{(16)}$.

Abdallah A.E.A et al revealed that PSV \& $\mathrm{SV}$ in control group of about $(2.27 \pm 0.94 \mathrm{~cm} / \mathrm{s} \&$ $27.26 \pm 2.05 \mathrm{ul})$ respectively ${ }^{(\mathbf{1 2})}$.

\section{In NPH patients:}

We reported obvious elevation of the PSV as well as the aqueductal SV in NPH patients in comparison to the control group with statistically significant difference ( $\mathrm{P}$ value of about 0.020 ), indicating hyper dynamic circulation. The SV in NPH patients was found higher with mean value 109.5 ( \pm 68.4) microliter compared to 27.6 ( \pm 2.3) microliter in normal control group with statistically significant difference $(\mathrm{P}$ value of about 0.015).Our results were compatible with Kahlon B et al who reported a statistical significant difference between NPH group\& control group. It was found also compatible with the study performed by ${ }^{(\mathbf{1})}$ on 20 cases (15 patients \& 5 controls) who reported markedly elevated PSV and SV values with statistically significant difference in comparison to healthy volunteers indicating hyper dynamic CSF flow ${ }^{(17)}$.

Ihab I. Baker et al also reported prominent flow void with marked elevation of PSV \& SV in comparison to healthy volunteers with mean values about $(9.1 \pm 3.1 \mathrm{~cm} / \mathrm{s} \& 141 \pm 83 \mathrm{ul} /$ cycle $)$ respectively ${ }^{(\mathbf{1 6})}$.

Senger KPS et al studied 72 patients (36 control \& $36 \mathrm{NPH}$ cases) \& reported elevation in PSV, ACSV \& other parameters with mean values of about $(8.12 \pm 2.53 \mathrm{~cm} / \mathrm{s} \& 152 \pm 49 \mathrm{ul})$ respectively, compared to $(3.99 \pm 1.56 \mathrm{~cm} / \mathrm{s}$ \& $32.1 \pm 12.3 \mathrm{ul})$ in normal control ${ }^{(8)}$.

\section{- In Cerebral atrophy patients:}

In our study, 6 patients who had clinical overlapping features of NPH and atrophy were diagnosed as atrophic dilatation, found to have evident lower PSV values of about $4.3( \pm 1.2)$ $\mathrm{cm} / \mathrm{s}$ compared to $5.4( \pm 0.3) \mathrm{cm} / \mathrm{s}$ of normal control \& lower aqueductal SV being about 16.2 
( \pm 7.4) ul compared to $27.6( \pm 2.3)$ ul in normal control group indicating a hypo dynamic CSF circulation and a diagnosis of atrophy. These results correlate well with the study of Schroder H.W.S et al ${ }^{(\mathbf{1 4})}$ \& Bradley WG et al ${ }^{(\mathbf{1 8})}$, which also found to be compatible with the study performed by Abdallah A.E.A et al who reported that In cerebral atrophy, blood flow to the brain is decreased; with markedly lower PSV and SV values in comparison to healthy volunteers ${ }^{(\mathbf{1 2})}$.

\section{Relation between Aq. Stroke volume (> 42) and shunt responsiveness in NPH patients:}

In our study, we found that at a cut off value for aqueductal SV of about $42 \mathrm{ul} /$ cycle, fourteen out of fifteen patients who had well response to shunt operation $(93.3 \%)$ had SV above 42 . While three out of four patients who had not responded to shunt operation $(75 \%)$ had SV between (28-42). Aqueductal stroke volume (> 42) shows sensitivity of about $(93.3 \%) \&$ specificity of about (75\%) with total accuracy of about $(89.5 \%)$ in predicting shunt responsiveness in NPH patients.

In our study, one NPH shunt responsive patient had SV between (28-42) suggestive of mild hyper dynamic CSF flow \& early NPH with positive LTT. So, we found that CSF stroke volume has a role in selection of patients into shunt responders (SV > 42) \& less likely responding $(\mathrm{SV}=28-42)$ but still has an opportunity of improving on shunt procedures\& still have a chance with CSF shunting \& should not be used as exclusion criteria.

In our study, one NPH shunt nonresponsive patient had SV above 42 with negative LTT, which is explained by the concomitant evident brain ischemic changes \& temporal lobe atrophy.

Bradley W.G. et al \& Kahlon B et al reported that patients who responded well to shunting for NPH have at least twice the aqueductal SV of healthy elderly patients (19) (17). Our results also correlate with other previous studies done by Bradley WG. et al ${ }^{(20)} \&$ Parkkola R.K et al ${ }^{(21)}$.

Bradley W.G. et al preoperatively studied 18 patients with a diagnosis of NPH. The threshold value for increased systolic SV was set at $42 \mu \mathrm{l}$. He also stated $80 \%$ sensitivity, $100 \%$ specificity, a $100 \%$ positive predictive value, and a $50 \%$ negative predictive value using this $\operatorname{method}^{(\mathbf{1 9})}$.

Bradley W.G. et al mentioned that the increased CSF SV is a more important predictor of shunt response than the increased CSF flow void sign, so Hyper dynamic CSF flow is thus an indirect, but easily measured sign of normal cerebral flow and shunt responsive NPH and help to diagnose \& differentiate NPH which may respond to shunt procedure from cerebral atrophy, as they do not respond to CSF diversion $^{(22)}$.

\section{Relation between LTT and shunt responsiveness in NPH patients:}

- $\quad$ Fourteen out of fifteen NPH patients who had well response to shunt operation had positive LTT $(93.3 \%)$.

- Four NPH patients who not responded to shunt were all negative to LTT $(100 \%)$.

- $\quad$ LTT shows sensitivity of about (93.3\%) \& specificity of about (100\%) with total accuracy of about $(94.7 \%)$ in predicting shunt responsiveness in NPH patients.

- One NPH shunt responsive patient had negative LTT with hyper dynamic CSF flow in PC MRI suggesting that negative LTT alone is not an exclusion criteria.

N.B: The six patients diagnosed with $A D$ had negative LTT.

Bradley W.G. et al (22) stated that, PC MRI is one of the tools of prediction of shunt responsiveness in NPH patients. Selection of ideal patients for shunting procedure is crucial. Favorable prognostic factors include: Short duration of symptoms (less than 6 months), short history of mental deterioration (only slight or 
moderate mental impairment), onset of gait abnormality preceding dementia, a known cause of communicating hydrocephalus (e.g., subarachnoid hemorrhage, meningitis) or noncommunicating hydrocephalus (aqueduct stenosis), temporary symptom relief after high volume lumbar puncture, especially gait, occurrence of B-waves during $50 \%$ or more of the recording time during continuous ICP monitoring, resistance to CSF outflow of 18 $\mathrm{mmHg} / \mathrm{ml}$ per minute or higher during continuous lumbar CSF infusion test, absence of significant cerebrovascular disease, aqueductal flow void on T2 weighted images and the pattern of hydrodynamic hydrocephalus and absence of substantial white matter lesions on MRI.

Ginera et al emphasized PC-MRI is a useful tool for the early diagnosis of patients with NPH \& prediction of shunt responsiveness ${ }^{(23)}$.

\section{Conclusion:}

PC MRI CSF flowmetry was found to be of high sensitivity and specificity in diagnosing NPH \& differentiating it from atrophic dilatation. It is simple, fast and non-invasive effective method that adds more to the total accuracy of the conventional MRI examination as it provide valuable additional information, increasing the confidence of the diagnosis, reducing rate of unnecessary previously used invasive techniques as ELD, thus reducing rates of complications and predicting shunt responsiveness.

\section{References:}

1. Hakim S, Adams RD. The special clinical problem of symptomatic hydrocephalus with normal cerebrospinal fluid: observations on cerebrospinal fluid hydrodynamics. J Neurol Sci; 2:307-327, 1965.
2. Brean A, Eide PK. Prevalence of probable idiopathic NPH in Norwegian population, Acta Neurol Scand.; 118; 48- 53, 2008.

3. Nagra G, Koh $L$, Aubert $I$, et al: Intraventricular injection of antibodies to beta1integrins generates pressure gradients in the brain favoring hydrocephalus development in rats. Am J Physiol Regul Integr Comp Physiol 297:R1312-R1321, 2009.

4. Siraj S: An overview of normal pressure hydrocephalus and its importance: how much do we really know? J Am Med Dir Assoc; 12(1): 19-21, 2011.

5. Ng SE, Low AM, Tang KK, Lim WE, Kwok RK. Idiopathic normal pressure hydrocephalus: correlating magnetic resonance imaging biomarkers with clinical response. Ann Acad Med Singapore.;38(9):803-8, 2009.

6. Etienne Holl, Neil D. Kitchen, Laurence D. Watkins: FRCS (SN). 2011.

7. Halperin JJ., Roger Kurlan: Practice guideline: Idiopathic normal pressure hydrocephalus: Response to shunting and predictors of response. American Academy of Neurology, 2015.

8. Senger KPS, Singh KR, Singh AK, et al: CSF flowmetry: an innovative technique in diagnosing normal pressure hydrocephalus. Int $\mathbf{J}$ Adv Med;4:682-7, 2017.

9. Bradley WG Jr: CSF flow in the brain in the context of normal pressure hydrocephalus. AJNR Am J Neuroradiol 36:831-838, 2015.

10. Nicole C. h. Keong, et al, FMedsci, Imaging normal pressure hydrocephalus: theories, techniques, and challenges, 10.3171/.7.FOCUS16194, 2016.

11. Bhadelia RA, Bogdan AR, Kaplan RF, et al. CSF pulsation amplitude and its quantitative relationship to cerebral blood flow pulsations: a phase-contrast MR flow imaging study. Neuroradiology;39:258-264, 1997.

12. Abdallah A. E. A, Shabaan M. H., Hassan M. A., Yassin A. N.; Giza/EG, The role of MRICSF flowmetry in differentiation between NPH and Involutional brain changes, 10.1594/ecr/C0117, 2015.

13. Rovira A., Capellades J., Grive I., Poca M.A., Pedraza S., Saherquillo J. And 
Rodriguezbaeza A.: $\quad$ Spontaneous ventriculostomy: Report of three cases reviewed by flow sensitive phase contrast cine MR imaging. American Journal of Neuroradiology, 20: 1647- 1652, 1999.

14. Schroder HW, Schweim C, Schweim KH, Gaab MR et al. Analysis of aqueductal cerebrospinal fluid flow after endoscopic aqueductoplasty by using cine phase-contrast magnetic resonance imaging. J Neurosurg;93:237-244, 2000.

15. Schroth G. And Klose U.: Pathological CSF pulsations. Neuroradiology, 35: 16-24, 1992.

16. Ihab I. Baker, Naglaa M. Elsayed, Mona M. El-Kalioubie, And Yasser A. Atta, Brain Atrophy Versus Normal Pressure Hydrocephalus by CSF Flow Measurement at MRI , Med. J. Cairo Univ., Vol. 77, No. 1, March: 19-26, 2009.

17. Kahlon B, Annertz M, Ståhlberg F, Rehncrona S. Is aqueductal stroke volume, measured with cine phase-contrast MRI scans useful in predicting outcome of shunt surgery in suspected normal pressure hydrocephalus? Neurosurgery.; 60(1): 124-9; discussion 9-30, 2007.

18. Bradley W.G.: MR prediction of shuntresponse in hydrocephalus: CSF morphology Vs. physiology. AJNR, 19: 1285-1287, 1998.
19. Bradley WG Jr, Scalzo D, Queralt J, Nitz WN, Atkinson DJ, Wong P. Normal-pressure hydrocephalus: evaluation with cerebrospinal fluid flow measurements at MR imaging. Radiology; 198:523-529, 1996.

20. Bradley WG, Safar FG, Furtado C, et al. Increased intracranial volume in normal pressure hydrocephalus: a clue to the etiology of "idiopathic" NPH? AJNR Am J Neuroradiol;25:1479-84, 2004.

21. Parkkola R.K., Komu M.E.S, Kotilainen E.M., Valtonen S.O., Thomsen C. And Gideon P.: Cerebrospinal fluid flow in patients with dilated ventricles studied with MR imaging. European Radiology, 2000.

22. Bradley WG, Scalzo D, Queralt J, et al . Normal-pressure hydrocephalus: evaluation with cerebrospinal fluid flow measurements at MR imaging. Radiology. ; 198(2): 523-529, 2000.

23. Ginera JF, Sanz-Requena R, Flórez N, Alberich-Bayarri A, García-Martía G, Ponz A, Martí-Bonmatí L: Quantitative phase contrast MRI study of cerebrospinal fluid flow: A method for identifying patients with normalpressure hydrocephalus. Neurología (Epub ahead of print), 2013.

Table (1): Socio-demographics:

\begin{tabular}{|c|c|c|c|c|c|c|c|}
\hline \multirow[t]{3}{*}{ Variable } & \multicolumn{2}{|c|}{$\begin{array}{c}\text { NPH } \\
(\mathbf{N}=19)\end{array}$} & \multicolumn{2}{|c|}{$\begin{array}{c}\text { AD } \\
(N=6)\end{array}$} & \multicolumn{2}{|c|}{$\begin{array}{c}\text { Control } \\
(\mathrm{N}=5)\end{array}$} & \multirow[t]{3}{*}{ P-value } \\
\hline & Mean & SD & Mean & SD & Mean & SD & \\
\hline & Median & Range & Median & Range & Median & Range & \\
\hline \multirow[t]{2}{*}{ Age (years) } & 63.7 & 7.2 & 71.0 & 3.2 & 55.8 & 3.3 & \multirow[t]{2}{*}{$0.002(\mathrm{~S})$} \\
\hline & 64.0 & $\begin{array}{c}(52.0- \\
80.0) \\
\end{array}$ & 70.0 & $\begin{array}{r}(68.0- \\
75.0) \\
\end{array}$ & 56.0 & $(51-60)$ & \\
\hline Variable & $\mathbf{N}$ & $\%$ & $\mathbf{N}$ & $\%$ & $\mathbf{N}$ & $\%$ & P-value \\
\hline \multicolumn{8}{|l|}{ Gender } \\
\hline Female & 6 & $31.6 \%$ & 3 & $50.0 \%$ & 3 & $60.0 \%$ & \multirow[t]{2}{*}{$0.439(\mathrm{NS})$} \\
\hline Male & 13 & $68.4 \%$ & 3 & $50.0 \%$ & 2 & $40.0 \%$ & \\
\hline
\end{tabular}


Table (2): Differences in PSV and Aq. Stroke volume according to study groups:

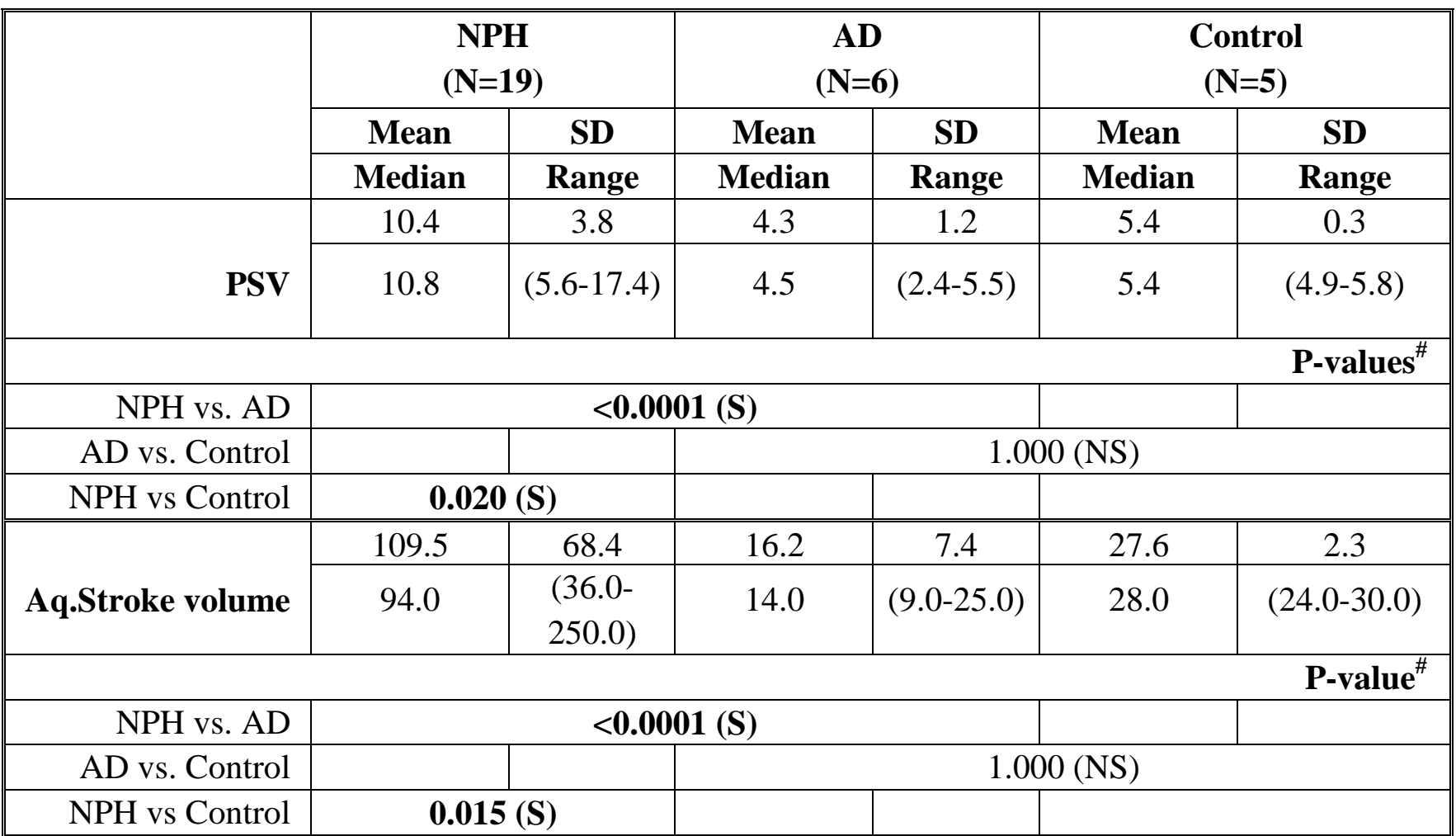

Table (3): Differences in PSV and Aq. Stroke volume in relation to shunt responsiveness in NPH patients:

\begin{tabular}{|c|c|c|c|c|c|}
\hline & \multicolumn{4}{|c|}{ Shunt responsiveness } & \multirow[t]{4}{*}{ P-value } \\
\hline & \multicolumn{2}{|c|}{$\begin{array}{c}\text { Well responsive } \\
(\mathrm{N}=15)\end{array}$} & \multicolumn{2}{|c|}{$\begin{array}{c}\text { Not responsive } \\
(\mathrm{N}=4)\end{array}$} & \\
\hline & Mean & SD & Mean & SD & \\
\hline & Median & Range & Median & Range & \\
\hline \multirow[b]{2}{*}{ PSV } & 11.4 & 3.5 & 6.6 & 1.8 & \multirow[b]{2}{*}{$0.006(\mathrm{~S})$} \\
\hline & 11.3 & $(5.7-17.4)$ & 5.7 & $(5.6-9.3)$ & \\
\hline \multirow[b]{2}{*}{ Aq. Stroke volume } & 125.9 & 67.4 & 48 & 21.4 & \multirow[b]{2}{*}{$0.009(\mathrm{~S})$} \\
\hline & 115.0 & $\begin{array}{l}(38.0- \\
250.0)\end{array}$ & 38.0 & $(36.0-80.0)$ & \\
\hline
\end{tabular}

Table (4): Relation between Aq. Stroke volume (> 42) and shunt responsiveness in NPH patients:

\begin{tabular}{|c|c|c|c|c|c|}
\hline \multirow[t]{3}{*}{ Variable } & \multicolumn{4}{|c|}{ "Shunt responsiveness } & \multirow[t]{3}{*}{ P-value $\#$} \\
\hline & \multicolumn{2}{|c|}{$\begin{array}{c}\text { Well responsive } \\
(\mathrm{N}=15)\end{array}$} & \multicolumn{2}{|c|}{$\begin{array}{c}\text { Not responsive } \\
(\mathrm{N}=4)\end{array}$} & \\
\hline & $\mathbf{N}$ & $\%$ & $\mathbf{N}$ & $\%$ & \\
\hline \multicolumn{6}{|c|}{ Aq. Stroke volume } \\
\hline$>42$ & 14 & $93.3 \%$ & 1 & $25.0 \%$ & \multirow[t]{2}{*}{$0.016(\mathrm{~S})$} \\
\hline $28-42$ & 1 & $6.7 \%$ & 3 & $75.0 \%$ & \\
\hline
\end{tabular}


Table (5): Relation between LTT and shunt responsiveness in NPH patients:

\begin{tabular}{|c|c|c|c|c|c|}
\hline \multirow[t]{3}{*}{ Variable } & \multicolumn{4}{|c|}{ Shunt responsiveness } & \multirow[t]{3}{*}{ P-value ${ }^{\#}$} \\
\hline & \multicolumn{2}{|c|}{$\begin{array}{l}\text { Well responsive } \\
\qquad(\mathrm{N}=15)\end{array}$} & \multicolumn{2}{|c|}{$\begin{array}{c}\text { Not responsive } \\
(N=4)\end{array}$} & \\
\hline & $\mathbf{N}$ & $\%$ & $\mathbf{N}$ & $\%$ & \\
\hline \multicolumn{6}{|l|}{ LTT } \\
\hline $\begin{array}{c}\text { Well } \\
\text { responsive }\end{array}$ & 14 & $93.3 \%$ & 0 & $0.0 \%$ & \multirow[t]{2}{*}{$0.001(S)$} \\
\hline $\begin{array}{c}\text { Not } \\
\text { responsive }\end{array}$ & 1 & $6.7 \%$ & 4 & $100.0 \%$ & \\
\hline
\end{tabular}

(Fig 1) Conventional MRI showing:

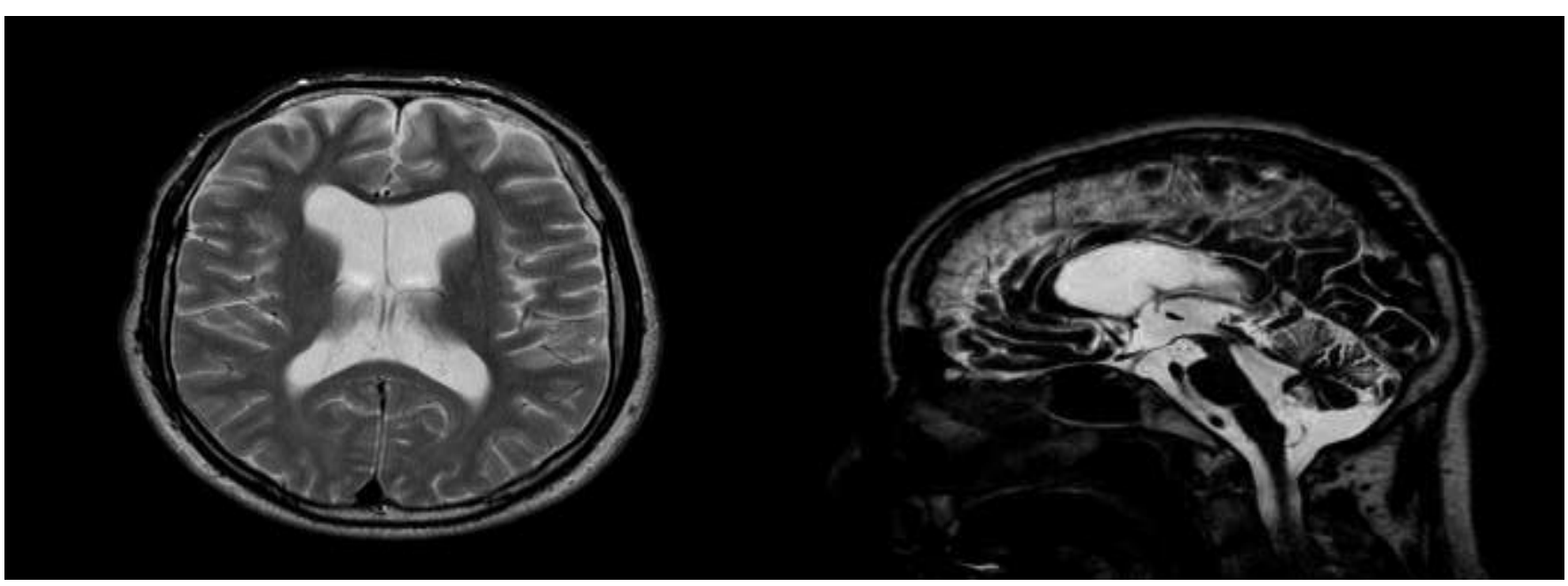

A. Axial T2

- Evident ventriculomegaly out of proportion of peripheral atrophic brain changes, marked corpus callosal thinning.

- Very limited peri-ventricular T2/FLAIR hyper intensity with mild ischemic changes.

- Prominent aqueductal flow void detected through the aqueduct of sylvius in CISS sequence.

- Mild Involutional changes.

(Fig 2) CSF flow study:

- Elevated CSF velocities and aqueductal systolic SV.

- $P S V=5.7 \mathrm{~cm} / \mathrm{s}$, Estimated systolic $S V=38$ microliter $/$ cycle.

\section{B. Sagittal T2}

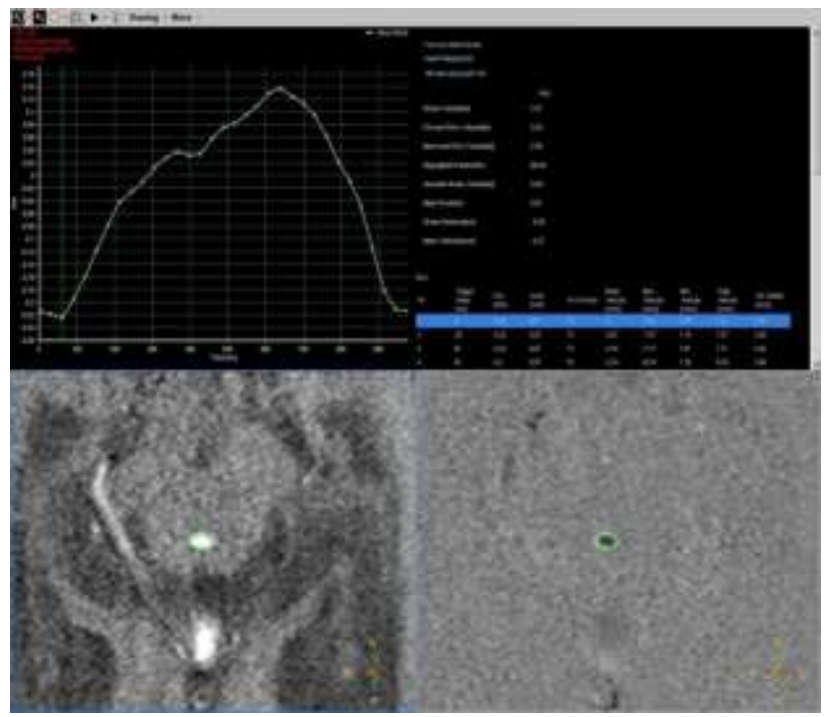




\section{(Fig 3) Conventional MRI showing:}

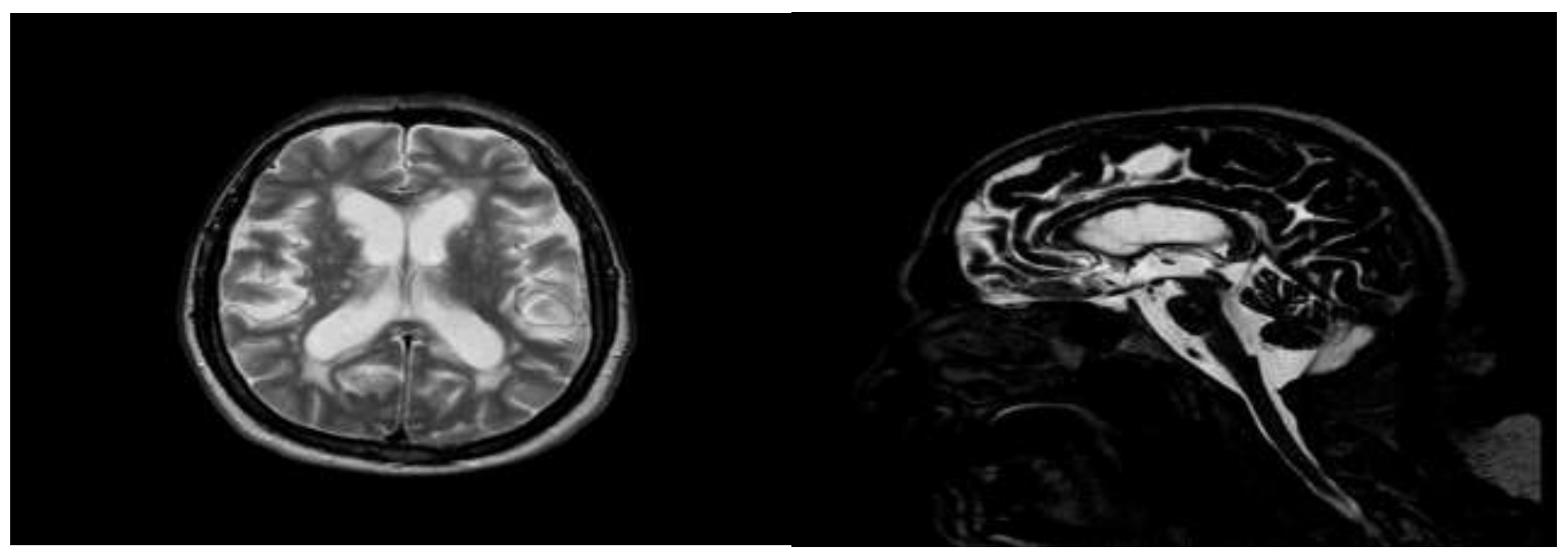

\section{Axial T2}

B. Sagittal T2

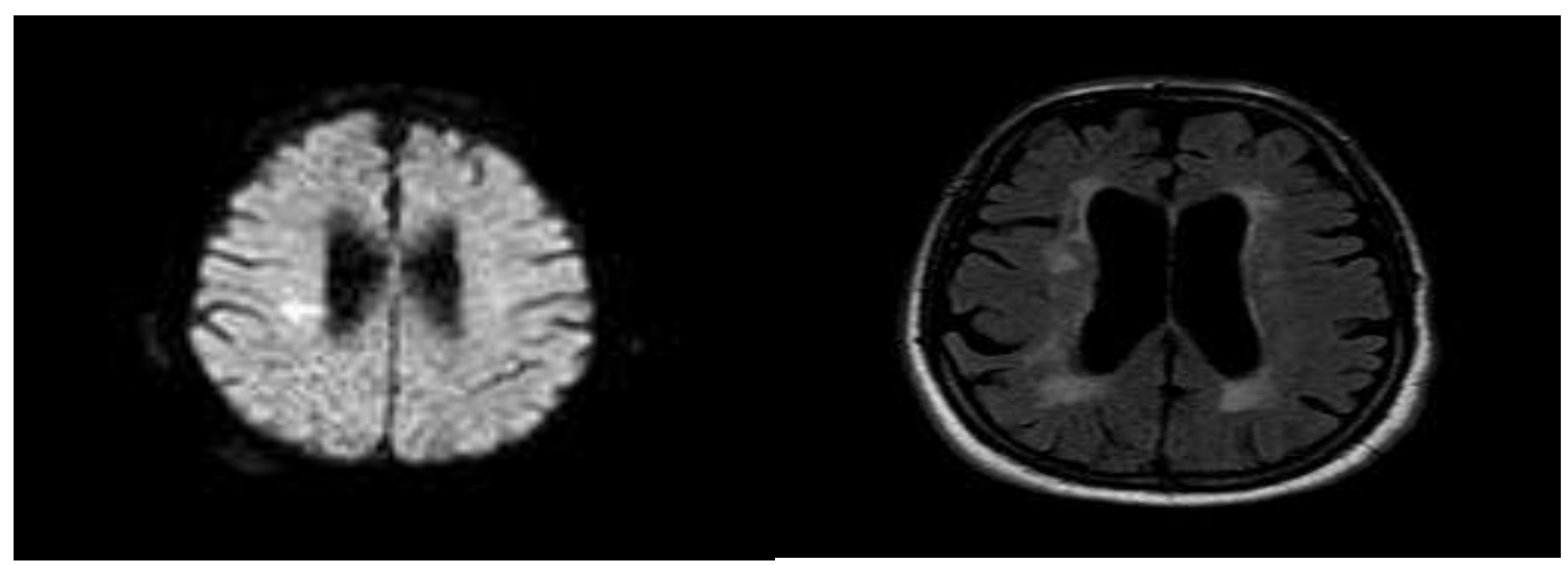

\section{E. Diffusion sequence}

D. FLAIR

- Evident dilatation of the supra and infra tentorial ventricular system.

- Bright T2 \& FLAIR signal are seen framing the periventricular white matter.

- Evident Involutional changes.

- Marked ischemic changes \& small vessel disease (Pontine and multiple bilateral periventricular lacunar infarctions with recent onset lacunes and diffuse subcortical arteriolo-leukoencephalopathy).

- Prominent aqueductal flow void detected through the aqueduct of sylvius in CISS sequence.

(Fig 4) CSF flow study:

- Markedly elevated CSF velocities and aqueductal SV.

- $P S V=9.27 \mathrm{~cm} / \mathrm{s}$, Estimated systolic $S V=80$ microlitre/cycle.

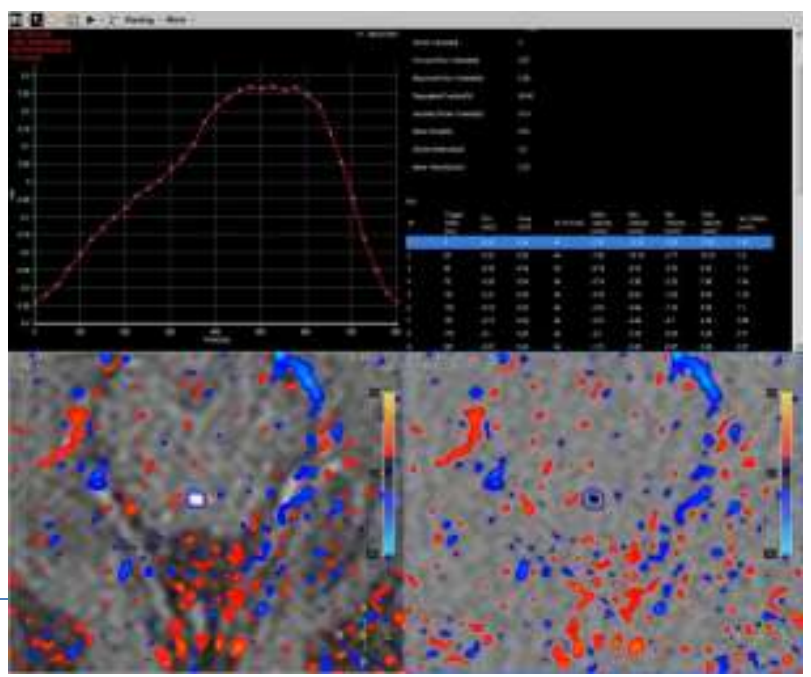

